\title{
Effect of antibiotic therapy on the prognosis of ventilator-associated pneumonia caused by Stenotrophomonas maltophilia
}

Bérénice Puech ${ }^{1 *}$ (D) Clémence Canivet ${ }^{1}$, Laura Teysseyre ${ }^{1}$, Guillaume Miltgen ${ }^{2,3}$, Thomas Aujoulat ${ }^{1}$, Margot Caron ${ }^{1}$, Chloé Combe ${ }^{1}$, Julien Jabot ${ }^{1}$, Olivier Martinet ${ }^{1}$, Jerome Allyn ${ }^{1,4}$, Cyril Ferdynus $^{4}$ and Nicolas Allou ${ }^{1,4}$

\begin{abstract}
Background: Ventilator-associated pneumonia (VAP) caused by Stenotrophomonas maltophilia is poorly described in the literature. However, it has been shown to be associated with increased morbidity and mortality. Probabilistic antibiotic therapy against S. maltophilia is often ineffective as this pathogen is resistant to many antibiotics. There is no consensus at present on the best therapeutic strategy to adopt (class of antibiotics, antibiotic combination, dosage, treatment duration). The aim of this study was to evaluate the effect of antibiotic therapy strategy on the prognosis of patients with VAP caused by S. maltophilia.

Results: This retrospective study evaluated all consecutive patients who developed VAP caused by S. maltophilia between 2010 and 2018 while hospitalized in the intensive care unit (ICU) of a French university hospital in Reunion Island, in the Indian Ocean region. A total of 130 patients with a median Simplified Acute Physiology Score II of 58 [43-73] had VAP caused by S. maltophilia after a median duration of mechanical ventilation of 12 [5-18] days. Ventilator-associated pneumonia was polymicrobial in $44.6 \%$ of cases, and ICU mortality was $50.0 \%$. After multivariate Cox regression analysis, the factors associated with increased ICU mortality were older age (hazard ratio (HR): 1.03; $95 \% \mathrm{Cl}$ $1.01-1.04, p=0.001)$ and high Sequential Organ Failure Assessment score on the day of VAP onset (HR: $1.08 ; 95 \% \mathrm{Cl}$ $1.03-1.14, p=0.002)$.

Appropriate antibiotic therapy, and in particular trimethoprim-sulfamethoxazole, was associated with decreased ICU mortality (HR: $0.42 ; 95 \% \mathrm{Cl} 0.24-0.74, p=0.003$ ) and decreased hospital mortality (HR: $0.47 ; 95 \% \mathrm{Cl} 0.28-0.79, p=0.04$ ). Time to start of appropriate antibiotic therapy, combination therapy, and duration of appropriate antibiotic therapy had no effect on ICU mortality $(p>0.5)$.
\end{abstract}

Conclusion: In our study, appropriate antibiotic therapy, and in particular trimethoprim-sulfamethoxazole, was associated with decreased ICU and hospital mortality in patients with VAP caused by S. maltophilia.

Keywords: Ventilator-associated pneumonia, Stenotrophomonas maltophilia, Outcome, Antibiotic therapy

*Correspondence: berenice.puech@gmail.com

1 Réanimation Polyvalente, Hôpital Universitaire Félix Guyon, Allée des Topazes, 97400 Saint Denis, France

Full list of author information is available at the end of the article

\begin{abstract}
Introduction
Ventilator-associated pneumonia (VAP) caused by Stenotrophomonas maltophilia is relatively common, with the latest report of the European Centre for Disease Prevention and Control stating that this microorganism is one of the 10 most frequently isolated germs in respiratory samples [1]. Depending on the study, the incidence $S$.
\end{abstract}


maltophilia in patients with VAP varies between from 0.3 and $2.0 \%[2,3]$. According to some authors, however, $S$. maltophilia is of limited pathogenicity and should not be considered as an infectious agent in the majority of VAP cases [4-6]. Admittedly, the link between colonization by S. maltophilia and respiratory infection is often difficult to establish. On the one hand, severely ill patients can be colonized with S. maltophilia in the respiratory tract without presenting respiratory symptoms $[4,5]$. On the other hand, half of respiratory samples containing strains of $S$. maltophilia show significant growth of other microorganisms with known pathogenicity [2, 7]. Several studies nonetheless suggest that VAP caused by $S$. maltophilia is associated with high morbidity and mortality $[3,4,7]$, in particular due to inappropriate probabilistic treatment $[7,8]$. In some studies, the mortality rate attributable to $S$. maltophilia colonization or infection was found to vary between 20.0 and $38.0 \%$ [8].

There is no consensus at present on the therapeutic strategy to adopt in cases of VAP caused by $S$. maltophilia (class of antibiotics, antibiotic combination, dosage, treatment duration). Some authors recommend high doses of trimethoprim-sulfamethoxazole based on clinical data, while others favor combination therapy based on in vitro data $[9,10]$. In view of this, our study aimed to evaluate the effect of antibiotic therapy strategy on the prognosis of patients with VAP caused by $S$. maltophilia.

\section{Methods}

This single-center retrospective study was conducted in the Multi-Purpose Intensive Care Unit (ICU) of Félix Guyon University Hospital in Reunion Island, a French overseas department located in the Indian Ocean.

All patients hospitalized in ICU with a blood or respiratory culture positive for S. maltophilia between 1 January 2010 and 31 December 2018 were evaluated and considered for inclusion. Patients aged over 18 years who developed VAP caused by $S$. maltophilia during their ICU stay were included in the study (Fig. 1).

In accordance with the French legislation on non-interventional studies [11], this study was registered with the National Institute of Health Data under the number MR 5611200420 and was approved by the Ethics Committee of the French Society of Infectious Disease and Tropical Medicine (CER-MIT 2021-0302). This study complies with the Strengthening the Reporting of Observational studies in Epidemiology recommendations statement [12].

\section{Definitions}

Ventilator-associated pneumonia caused by $S$. maltophilia was defined by: new or progressive infiltrate; temperature $>38.0{ }^{\circ} \mathrm{C}$ or $<36.5^{\circ} \mathrm{C}$; white blood cell count $>12 \mathrm{G} / \mathrm{L}$ or $<4 \mathrm{G} / \mathrm{L}$; purulent secretions; drop in oxygenation; positive respiratory culture obtained by bronchoalveolar lavage (threshold: $10^{4}$ colony forming unit (CFU)/ $\mathrm{mL}$ ), protected distal sampling (threshold: $10^{3} \mathrm{CFU} / \mathrm{mL}$ ) or endotracheal aspiration (threshold: $10^{6} \mathrm{CFU} / \mathrm{mL}$ ); and occurrence of infection $48 \mathrm{~h}$ or more after tracheal intubation. [13].

All patients with a Clinical Pulmonary Infection Score (CPIS) $>6$ and those with a CPIS $\leq 6$ who were treated by a clinician for VAP were evaluated [14].

\section{Pharmacological management}

In accordance with our protocol, all patients with VAP caused by $S$. maltophilia were treated with: trimethoprim-sulfamethoxazole $(1200 \mathrm{mg} / 240 \mathrm{mg}) / 6 \mathrm{~h}$ and/or ciprofloxacin $400 \mathrm{mg} / 8 \mathrm{~h}$ and/or moxifloxacin and/or ticarcillin-clavulanate $4 \mathrm{~g} / 8 \mathrm{~h}$ and/or ceftazidime $2 \mathrm{~g} / 6 \mathrm{~h}$.

Dosages were adjusted to renal function if necessary, and the choice of antibiotics was left to the discretion of the clinician.

\section{Data collection}

The following information was collected:

- Demographic data (age, sex), organ failure during ICU stay and on the day of VAP onset, and comorbidities [diabetes, hypertension, chronic heart failure, chronic respiratory failure, chronic liver failure, chronic renal failure, immunodeficiency, recent or ongoing chemotherapy, chronic alcohol abuse, body mass index (BMI) $>30 \mathrm{~kg} / \mathrm{m}^{2}$, malnourishment (BMI $<18.5 \mathrm{~kg} / \mathrm{m}^{2}$ or weight loss $>10 \%$ over the previous 6 months)].

- Use of extracorporeal organ support such as extracorporeal membrane oxygenation and/or renal replacement therapy, use of catecholamines, mechanical ventilation (MV) settings, Glasgow score.

- Highest bilirubin level, lowest platelet level, lowest prothrombin level, and lowest $\mathrm{PaO} 2 / \mathrm{FiO} 2$ ratio during ICU stay and $\mathrm{PaO} 2 / \mathrm{FiO} 2$ ratio on the day of VAP onset.

- Simplified Acute Physiology Score II (SAPS II) within $48 \mathrm{~h}$ of admission and Sequential Organ Failure Assessment (SOFA) scores on admission and on the day of VAP onset.

- Data on infection: type of respiratory sample, type of infection (monomicrobial or polymicrobial), duration of previous MV, known rectal or respiratory colonization with S. maltophilia, and CPIS on the day of microbiological sampling.

- Susceptibility of S. maltophilia strains to selected antimicrobial agents, as determined using the disk- 


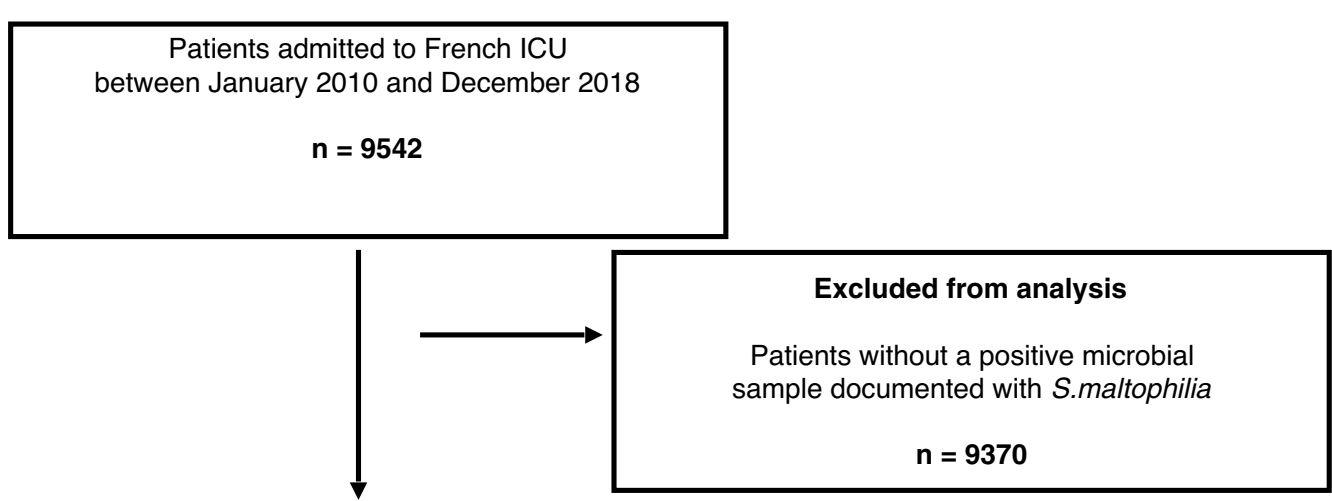

Patients with a positive S.maltophilia blood and/or respiratory culture during their ICU stay

$$
n=172
$$

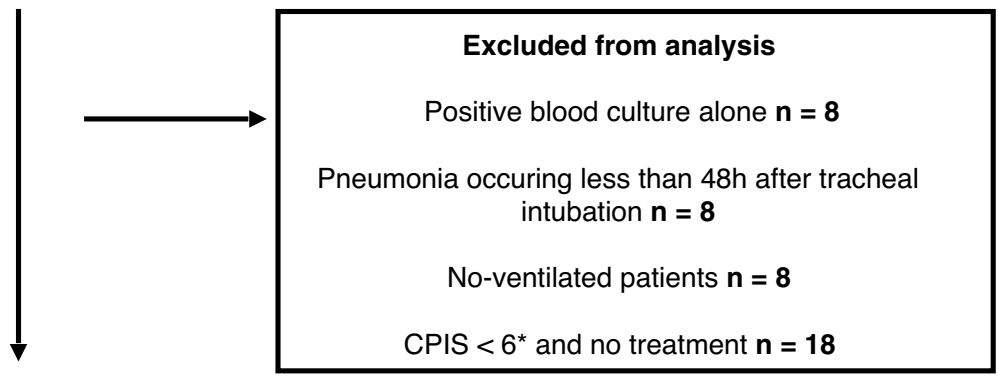

Patients with a documented S.maltophilia considered for final analysis

$$
n=130
$$

Fig. 1 Flowchart

diffusion method and the minimum inhibitory concentrations defined by the European Committee on Antimicrobial Susceptibility Testing [15].

- Data on treatment of VAP caused by S. maltophilia: class of antibiotics, type of therapy (monotherapy or combination therapy), as well as time to start and duration of appropriate antibiotic therapy.

- Prognosis data: MV duration, length of stay in ICU and in hospital, ICU and hospital mortality.

\section{Statistical analyses}

Patient characteristics were described as frequency and percentage for categorical variables and as median and interquartile range for quantitative variables. Qualitative variables were compared using the Chi-square test, the Kruskal-Wallis test or Fisher's exact test, as appropriate. Continuous variables were compared using the nonparametric Mann-Whitney test. Bivariate analysis of the main outcome (ICU mortality) was performed using the Kaplan-Meier method and comparisons were performed using the log-rank test. A multivariate analysis was conducted with a Cox model. A competing risk analysis of the main outcome was also performed for patients who underwent Withholding of Life-Sustaining Treatment (WLST) versus those who did not. Adjusted hazard ratios (HR) and their 95\% confidence intervals (CI) were calculated. The proportional risk hypothesis was tested 
by introducing an interaction between groups (treated patients versus non-treated patients) and time. All tests were performed at a 2-tailed type I error of $5 \%$ using SAS 9.4 software (SAS Institute Inc., Cary, NC).

\section{Results}

\section{Population}

Between January 2010 and December 2018, 9542 patients were hospitalized in our ICU, 130 (1.4\%) of whom presented with VAP caused by S. maltophilia. Our study population was predominantly male (63.8\%), with a median age of 61 [51-70] years. Median severity scores on admission were 9 [7-12] for SOFA and 58 [43-73] for SAPS II. Reasons for admission to ICU were acute respiratory failure in almost half of cases (48.5\%), followed by septic shock (23.1\%), post-operative management of cardiac surgery (21.5\%), neurological causes, cardiogenic shock, and polytrauma. Lastly, $36.2 \%$ of our patients had chronic heart failure, $20.8 \%$ had chronic respiratory failure, $30.7 \%$ had chronic alcohol abuse, and $30.7 \%$ were malnourished (Table 1).

\section{Sampling methods and microorganism distribution}

Respiratory samples were obtained through endotracheal aspiration in $46.2 \%$ of cases, protected distal sampling in $34.6 \%$ of cases, and bronchoalveolar lavage in $19.2 \%$ of cases.
Infection was polymicrobial in $44.6 \%$ of cases. The microorganism most frequently associated with $S$. maltophilia was Pseudomonas aeruginosa (Table 3 ).

Median CPIS was 8 [7-9], and MV duration before onset of VAP was 12 [5-18] days (Table 2).

\section{Susceptibility of Stenotrophomonas maltophilia strains and type of antibiotic therapy}

Identified strains of $S$. maltophilia were susceptible to trimethoprim-sulfamethoxazole in $86.2 \%$ of cases, to fluoroquinolones in $85.4 \%$ of cases, to ticarcillin-clavulanate in $58.5 \%$ of cases, and to ceftazidime in $40.0 \%$ of cases. Appropriate antibiotic therapy was initiated after a median of 2 [1-3] days.

Dual antibiotic therapy was initiated in $53(40.8 \%)$ patients, $43(81.1 \%)$ of whom received trimethoprimsulfamethoxazole and fluoroquinolone. Triple antibiotic therapy was initiated in 35 (26.9\%) patients, 32 (91.4\%) of whom received ticarcillin-clavulanate, trimethoprim-sulfamethoxazole, and fluoroquinolone (Table 3). A total of 38 patients (29.2\%) received no treatment for $S$. maltophilia. There was no significant difference between treated and untreated patients with respect to the CPIS $(p=0.17)$, the SOFA score on the day of VAP onset $(p=0.43)$, the polymicrobial character of the infection $(p=0.12)$, or the presence of WLST $(p=0.55)$. Probabilistic antibiotic therapy was effective against the co-infecting microorganism(s) in $86.2 \%$ of cases $(50 / 58)$ and in

Table 1 Demographic and baseline characteristics of ICU patients with VAP caused by S. maltophilia

\begin{tabular}{|c|c|c|c|c|}
\hline Variables & Total $N=130$ & $\begin{array}{l}\text { Alive at ICU discharge } \\
n=65\end{array}$ & $\begin{array}{l}\text { Dead at ICU discharge } \\
n=65\end{array}$ & $p$-value \\
\hline Sex, male & $83(63.8)$ & $41(63.1)$ & $42(64.6)$ & 0.85 \\
\hline Sex, female & $47(36.2)$ & $24(18.5)$ & $23(35.4)$ & \\
\hline Age, years & $61[51-70]$ & $56[44-65]$ & $63[56-65]$ & $<0.001$ \\
\hline \multicolumn{5}{|l|}{ Comorbidities } \\
\hline Chronic respiratory failure & $27(20.8)$ & $10(15.4)$ & $17(26.2)$ & 0.13 \\
\hline Chronic heart failure & $47(36.2)$ & $18(27.7)$ & $29(44.6)$ & 0.04 \\
\hline Chronic renal failure & $30(23.1)$ & $9(13.8)$ & $21(32.3)$ & 0.01 \\
\hline Immunodeficiency & $6(4.6)$ & $2(3.1)$ & $4(6.2)$ & 0.68 \\
\hline Hypertension & $64(49.2)$ & $27(41.5)$ & $37(56.9)$ & 0.08 \\
\hline Recent or ongoing chemotherapy & $12(9.2)$ & $4(6.1)$ & $8(12.3)$ & 0.22 \\
\hline $\mathrm{BMI}>30 \mathrm{~kg} / \mathrm{m}^{2}$ & $29(22.3)$ & $14(21.5)$ & $15(23.1)$ & 0.83 \\
\hline Malnourishment & $40(30.7)$ & $20(30.8)$ & $20(30.8)$ & 1 \\
\hline Diabetes & $42(32.3)$ & $15(23.1)$ & $27(41.5)$ & 0.002 \\
\hline \multicolumn{5}{|l|}{ Severity scores } \\
\hline SOFA score on admission & $9[7-12]$ & $9[7-11]$ & $10[7-12]$ & 0.06 \\
\hline SAPS $\|$ & $58[43-73]$ & $50[37-68]$ & $65[50-76]$ & 0.003 \\
\hline SOFA score on day of VAP onset & $7[4-11]$ & $6[3-9]$ & $10[6-12]$ & $<0.001$ \\
\hline
\end{tabular}

Quantitative variables are expressed as median [25-75th percentiles] and qualitative variables as number (\%) 
Table 2 Description and treatment of VAP caused by S. maltophilia

\begin{tabular}{|c|c|c|c|c|}
\hline Variables & Total $N=130$ & $\begin{array}{l}\text { Alive at ICU } \\
\text { discharge } n=65\end{array}$ & $\begin{array}{l}\text { Dead at ICU } \\
\text { discharge } n=65\end{array}$ & $p$-value \\
\hline \multicolumn{5}{|l|}{ Infection } \\
\hline Bacteremia & $4(3.1)$ & $1(1.5)$ & $3(4.6)$ & 0.89 \\
\hline Polymicrobial & $58(44.6)$ & $32(49.2)$ & $26(40)$ & 0.29 \\
\hline Duration of mechanical ventilation before onset of VAP, days & $12[5-18]$ & $13.5[5.5-19]$ & $10[5-16]$ & 0.62 \\
\hline \multicolumn{5}{|l|}{ Treatment } \\
\hline No treatment & $38(29.2)$ & $18(13.8)$ & $20(15.4)$ & 0.57 \\
\hline Monotherapy & $4(0.03)$ & $3(0.02)$ & 1 & \\
\hline Combination therapy $(\geq 2)$ & $88(67.7)$ & $44(67.7)$ & $44(67.7)$ & \\
\hline Trimethoprim/sulfamethoxazole & $80(62.5)$ & $40(62.5)$ & $40(62.5)$ & 1 \\
\hline Fluoroquinolone & $84(65.6)$ & $40(62.5)$ & $44(68.7)$ & 0.46 \\
\hline Ticarcillin-clavulanate & $39(30.4)$ & $20(31.3)$ & $19(29.7)$ & 0.85 \\
\hline Ceftazidime & $9(7.0)$ & $5(7.8)$ & $4(6.3)$ & 0.99 \\
\hline Duration of appropriate antibiotic therapy, days & $8[5-14]$ & $9[6-14]$ & $7[3-12]$ & 0.12 \\
\hline Time to start of appropriate antibiotic therapy, days & $2[1-3]$ & $2[1,2]$ & $2[1-3]$ & 0.58 \\
\hline
\end{tabular}

Quantitative variables are expressed as median [25-75th percentiles] and qualitative variables as number (\%)

$V A P$ ventilator-associated pneumonia, ICU intensive care unit, IQR interquartile range

Table 3 Microbiological description of ventilator-associated pneumonia caused by S. maltophilia

\begin{tabular}{ll}
\hline Variables & Total $\mathbf{N}=\mathbf{1 3 0}$ \\
\hline Monomicrobial (S. maltophilia) & $72(55.4)$ \\
Polymicrobial & $58(44.6)$ \\
Acinetobacter spp. & 4 \\
Citrobacter spp. & 1 \\
Enterobacter spp. & 10 \\
Enterococcus spp. & 5 \\
Escherichia coli & 2 \\
Klebsiella spp. & 5 \\
Proteus spp. & 4 \\
Pseudomonas aeruginosa & 24 \\
Serratia spp. & 3 \\
Staphylococcus spp. & 6 \\
Other & 1 \\
\hline
\end{tabular}

Qualitative variables are expressed as number (\%)

$100 \%$ of cases when the co-infecting microorganism was P. aeruginosa.

\section{Prognosis and risk factors associated with ICU mortality}

Median MV duration was 21 [14-37] days and median MV duration after onset of VAP was 7 [4-15] days. Tracheotomy was performed in $31.5 \%$ of patients, with a statistically higher incidence in surviving patients $[41.5 \%$ in survivors versus $21.5 \%$ in non-survivors $(p=0.01)]$. ICU mortality was $50.0 \%$ and hospital mortality was $56.2 \%$.
The Kaplan-Meier method using the log-rank test found a significant difference in ICU survival and hospital survival between treated and non-treated patients ( $p=0.009$ and $p=0.02$, respectively) (Fig. 2). The only antibiotic therapy associated with a significant difference between treated and non-treated patients was trimethoprim-sulfamethoxazole $(p=0.02)$ (Fig. 3$)$. For the others, there was no difference between treated and non-treated patients: fluoroquinolones $(p=0.54)$, ticarcillin-clavulanate $(p=0.14)$, and ceftazidime $(p=0.64)$.

After adjustment for confounding factors (Table 4), appropriate antibiotic therapy was associated with decreased ICU mortality (HR: 0.42; 95\% CI 0.24-0.74, $p=0.003$ ) and decreased hospital mortality (HR: 0.47; 95\% CI 0.28-0.79, $p=0.04)$. When taking competing risks into consideration (Table 5), appropriate antibiotic therapy was associated with a similar significant decrease in ICU mortality in patients who underwent WLST (HR: 0.47; 95\%CI 0.24-0.90; $p=0.02$ ) and in patients who did not (HR: 0.33; 95\% CI 0.11-0.97; $p=0.04$ ).

\section{Discussion}

We performed a search of the literature in English and French using the terms pneumonia, S. maltophilia, intensive care unit, and outcome. We found three articles on VAP caused by $S$. maltophilia, two of which were conducted in adult populations. The first was the multicenter study by Guerci et al. (236 cases) and the second the study by Ibn Saied et al. (102 cases from the OUTCOMEREA database) $[2,16]$. Both studies examined the impact of therapeutic modalities (antibiotic combination, 

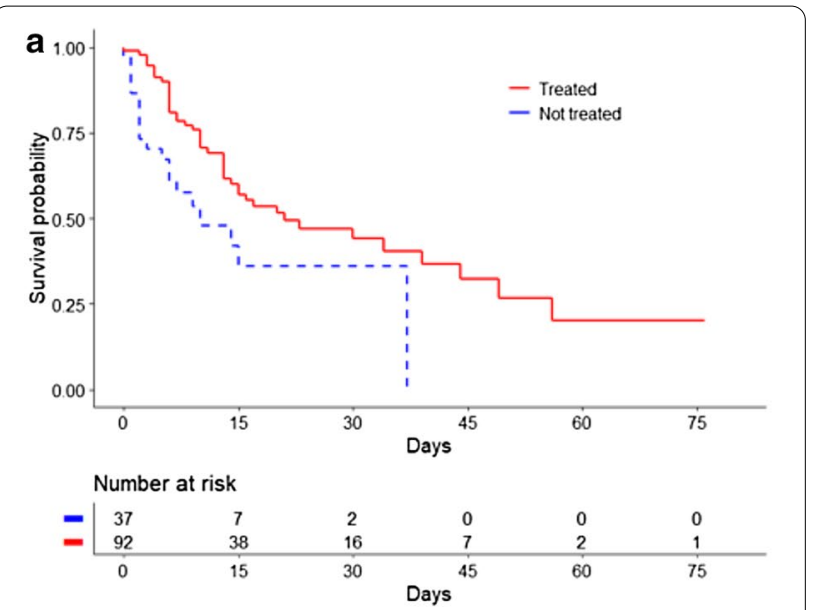

b

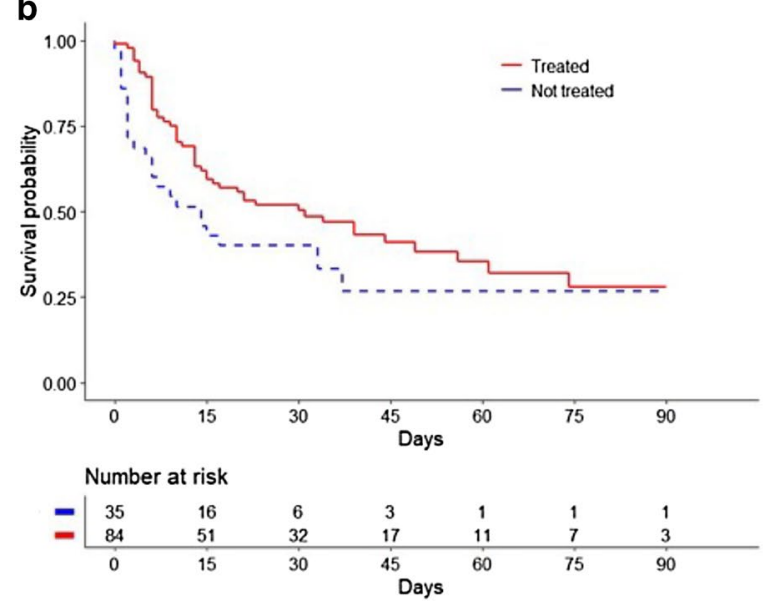

Fig. 2 Kaplan-Meier estimates of the probability of survival. a ICU Survival according to an appropriate antibiotic treatment against $S$. maltophilia. b Hospital survival according to an appropriate antibiotic treatment against $S$. maltophilia

treatment duration, etc.) on the prognosis of patients, but neither of them evaluated the association between prognosis and lack of appropriate treatment.

As in other studies of VAP caused by S. maltophilia [4, 16], the risk factors associated with increased ICU mortality were older age and high SOFA score on the day of VAP onset.

An original finding of our study was that appropriate antibiotic therapy, and in particular trimethoprim-sulfamethoxazole, is associated with decreased ICU mortality (HR: 0.42; 95\% CI 0.24-0.74, $p=0.003$ ) and hospital mortality (HR: $0.47 ; 95 \%$ CI $0.28-0.79, p=0.04$ ). However, time to start of appropriate antibiotic therapy and duration of appropriate antibiotic therapy had no effect on mortality.

Most studies of ICU patients with pneumonia caused by $S$. maltophilia found no association between mortality and antibiotic therapy strategy $[2,16]$. The only exceptions are the study by Tseng et al. [17] and Hanes et al. [7], in which mortality was associated with delayed initiation of appropriate antibiotic therapy. We found no such association in our study, even though appropriate antibiotic therapy was initiated 2 days after VAP onset. The discrepancy between our results and those of Hanes et al. may be partly explained by the fact that the latter found a higher rate of co-infection in their cohort, and in particular a higher rate of co-infection with $P$. aeruginosa $(92.3 \%$ of patients with a co-infection and $34.6 \%$ with a co-infection with $P$. aeruginosa versus $44.6 \%$ with a co-infection and $18.5 \%$ with a co-infection with $P$. aeruginosa in our study) [7]. Likewise, in the study by Tseng et al., the association between mortality and delayed initiation of appropriate antibiotic therapy was especially strong in cases of co-infection [17]. In line with these findings, $Y$ in et al. observed that co-infection with $P$. aeruginosa, which is common in cases of $S$. maltophilia pneumonia, is a poor prognostic factor [18]. In our study, however, the rate of co-infection was the same in survivors and non-survivors $(p=0.29)$.

Studies examining VAPs caused by non-fermenting Gram-negative bacilli (GNB) found no effect of treatment duration on mortality, MV duration, or length of stay in ICU. However, in the randomized prospective study by Chastre et al., prolonged antibiotic therapy (15 days) was associated with fewer relapses than short antibiotic therapy ( 8 days) in the subgroup of patients infected with non-fermenting GNB (41.0\% of relapses in patients who received 8 days of treatment versus $25.0 \%$ in patients who received 15 days of treatment) [19]. Similarly, in a systematic review of prolonged treatment for hospitalacquired pneumonia, Pugh et al. observed fewer relapses in patients infected with non-fermenting GNB who had received prolonged treatment (OR: 2.18; 95\% CI 1.1-4.2) [20]. It should be noted, however, that the most frequently implicated non-fermenting GNB in the evaluated studies was $P$. aeruginosa $[19,20]$. A randomized study is currently underway that evaluates the effect of treatment duration on the prognosis of patients with VAP caused by P. aeruginosa [21].

In our study, patients were treated mainly with combination therapy, with no difference in effect on mortality between dual and triple therapy. The difference in effect on mortality between combination therapy and monotherapy could not be evaluated, as only 4 of our patients received monotherapy. Our study suggests that trimethoprim-sulfamethoxazole has a protective effect in patients with VAP caused by $S$. maltophilia. This finding is in line with other studies of patients with S. maltophilia pneumonia, in which trimethoprim-sulfamethoxazole was not associated with excess mortality or the emergence of resistance, even when used as monotherapy [2, 22-24]. 

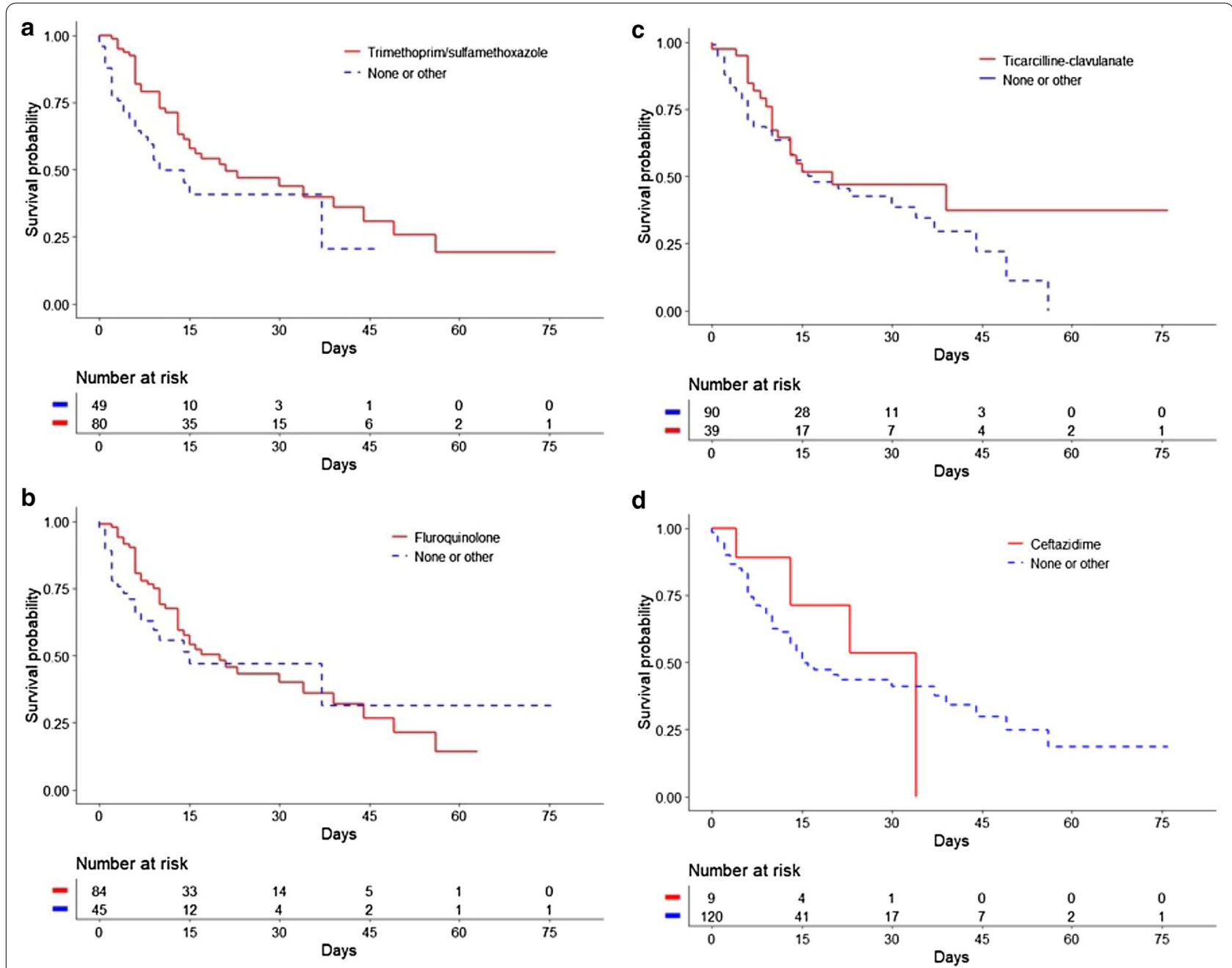

Fig. 3 Kaplan-Meier estimates of the probability of ICU Survival. a ICU survival according to the antibiotic treatment with trimethoprim/ sulfamethoxazole. $\mathbf{b}$ ICU survival according to antibiotic treatment with fluoroquinolone. $\mathbf{c}$ ICU survival according to antibiotic treatment with ticarcillin/clavulanate. $\mathbf{d}$ ICU survival according to antibiotic treatment ceftazidime

Table 4 Risk factors independently associated with ICU mortality in the 130 patients assessed by multivariate Cox regression analysis

\begin{tabular}{lll}
\hline Variables & Hazard ratio (Cl 95\%) & $\boldsymbol{p}$-value \\
\hline Appropriate treatment & $0.42(0.24-0.74)$ & 0.003 \\
Age & $1.03(1.01-1.04)$ & 0.003 \\
SOFA score on day of VAP onset & $1.07(1.02-1.13)$ & 0.006
\end{tabular}

Qualitative variables are expressed as number (\%)

$I C U$ intensive care unit, $\mathrm{Cl}$ confidence interval, SOFA Sequential Organ Failure Assessment, VAP ventilator-associated pneumonia

One in vitro study did find that combination therapy broadens the spectrum of antibiotics and constitutes as such a more effective probabilistic treatment for patients with sepsis, particularly in cases of non-fermenting GNB
Table 5 Competing risk factors independently associated with ICU mortality in the 130 patients assessed by multivariate Cox regression analysis

\begin{tabular}{lll}
\hline Variables & Hazard ratio (Cl 95\%) & $\boldsymbol{p}$-value \\
\hline Death without WLST & & \\
$\quad$ Appropriate treatment & $0.33(0.11-0.97)$ & 0.04 \\
Age & $1.02(0.98-1.05)$ & 0.35 \\
$\quad$ SOFA score on day of VAP onset & $1.12(1.01-1.24)$ & 0.04 \\
Death with WLST & & \\
$\quad$ Appropriate treatment & $0.47(0.24-0.90)$ & 0.02 \\
$\quad$ Age & $1.03(1.01-1.05)$ & 0.004 \\
$\quad$ SOFA score on day of VAP onset & $1.12(1.01-1.24)$ & 0.05 \\
\hline
\end{tabular}

Qualitative variables are expressed as number (\%)

Cl confidence interval, WLST Withholding of Life-Sustaining Treatment, SOFA Sequential Organ Failure Assessment, VAP ventilator-associated pneumonia 
infection [25]. However, clinical studies found no superiority of combination therapy over monotherapy in cases of sepsis, whether in terms of clinical cure or mortality, and this even in cases of non-fermenting GNB infection $[26,27]$.

The incidence of VAP caused by S. maltophilia was relatively high (1.4\%) in our ICU compared to what has been reported in the literature. Ibn Saied et al. and Guerci et al. reported an incidence of $0.5 \%$ and $0.3 \%$, respectively-keeping in mind that the latter study examined all cases of hospital-acquired S. maltophilia pneumonia $[2,16]$. The study by Nseir et al. found an incidence of $2.0 \%$, but it included cases of colonization with $S$. maltophilia in addition to infection cases [3]. The discrepancy between these and our results may be explained by the fact that our study was conducted in a tropical environment, which has been shown to favor the development of the parent microorganism Acinetobacter baumannii [28]. Another potential explanation is that our patients were diagnosed using the highly sensitive sampling technique of endotracheal aspiration.

In our study, ICU mortality was $50.0 \%$ and hospital mortality was $56.2 \%$. These findings are in line with the study by Saugel et al., in which ICU mortality was $50.0 \%$ [4]. While Guerci et al. found a hospital mortality of $50.0 \%$, their study included all cases of hospital-acquired pneumonia caused by S. maltophilia and not just those of VAP [2]. The study by Ibn Saied et al., in which the median SOFA score on the day of VAP onset was the same as in our study, found an ICU mortality of $40.0 \%$ despite the fact that $68.0 \%$ of patients received no treatment [16]. It may be that Ibn Saied et al. included cases of colonization with $S$. maltophilia in addition to cases of infection in their sample, which could explain this lower ICU mortality [16]. The study by Saugel et al., in which ICU mortality was $29.0 \%$ in colonized patients, would tend to support this hypothesis [4].

Our study has some limitations. The single center and retrospective nature of the study may have led to biases. In addition, the relatively small size of our sample limits the statistical power of the results. The size of our sample stems from our decision to exclude patients with a CPIS $\leq 6$ who were not treated by a clinician for VAP, as we assumed these to be cases of colonization with $S$. maltophilia or ventilator-associated tracheobronchitis $[4,29]$. It should be noted, however, that the retrospective studies by Guerci et al. and Ibn Saied et al. included a comparable number of patients: 228 ( $80.0 \%$ of whom had VAP) and 102 , respectively $[2,16]$.

Another limitation of our study is that we did not collect data on relapse or emergence of resistance. Lastly, we used mostly endotracheal aspiration with quantitative cultures, which may have led us to overestimate the incidence of S. maltophilia in our population due to heightened sensitivity. Current guidelines for the management of VAP allow the use of endotracheal aspirates (in addition to invasive respiratory specimens), but with semi-quantitative as opposed to quantitative cultures. In France, however, endotracheal aspiration with quantitative cultures is the most commonly used technique for the diagnosis of VAP [30]. Thus, in the study by Guerci et al., which represents the largest sample of patients with VAP caused by S. maltophilia to date, $30.0 \%$ of cases were diagnosed using this technique [2].

\section{Conclusion}

This is the third retrospective study of VAP caused by $S$. maltophilia to use a large sample of patients and the first to focus primarily on the effect of antibiotic therapy on the prognosis of infected patients.

In our study, appropriate antibiotic therapy, and in particular trimethoprim-sulfamethoxazole, was associated with decreased ICU and hospital mortality in patients with VAP caused by $S$. maltophilia.

\section{Abbreviations \\ VAP: Ventilator-associated pneumonia; ICU: Intensive care unit; CFU: Colony forming unit; CPIS: Clinical Pulmonary Infection Score; BMI: Body mass index; MV: Mechanical ventilation; SAPS II: Simplified Acute Physiology Score II; SOFA: Sequential Organ Failure Assessment; HZ: Hazard ratio; Cl: Confidence interval; WLST: Withholding of Life-Sustaining Treatment; GNB: Gram-negative bacilli.}

\section{Acknowledgements}

Not applicable.

\section{Authors' contributions}

$\mathrm{BP}, \mathrm{NA}$, and CF had full access to all of the data in the study and take responsibility for the integrity of the data and the accuracy of the data analysis. Study concept and design: BP and NA; acquisition of data: BP and CC; analysis and interpretation of data: BP, NA, CF and JA; drafting of the manuscript: BP, NA, and Jj; critical revision of the manuscript for important intellectual content: NA and JJ; statistical analysis: NA and CF; administrative, technical, or material support: BP, NA, CC, LT, CC, MC, and TA; study supervision: BP and NA. All authors read and approved the manuscript.

Funding

Not applicable.

Availability of data and materials

The datasets used and/or analyzed for this study are available from the corresponding author on reasonable request.

\section{Declarations}

Ethics approval and consent to participate

The study was approved by the Ethics Committee of the French Society of Infectious Disease and Tropical Medicine (CER-MIT 2021-0302) and was registered with the National Institute of Health Data under the number MR 5611200420.

\section{Consent for publication}

Not applicable.

Competing interests

The authors declare that they have no competing interests. 


\section{Author details}

${ }^{1}$ Réanimation Polyvalente, Hôpital Universitaire Félix Guyon, Allée des Topazes, 97400 Saint Denis, France. ${ }^{2}$ Service de Microbiologie, Hôpital Universitaire Félix Guyon, Allée des Topazes, 97400 Saint Denis, France. ${ }^{3}$ UMR Processus Infectieux en Milieu Insulaire Tropical (PIMIT), CNRS 9192, INSERM U1187, IRD 249, Université de La Réunion, Saint-Denis, France. ${ }^{4}$ Département d'Informatique Clinique, Hôpital Universitaire Félix Guyon, Allée des Topazes, 97400 Saint Denis, France.

Received: 4 June 2021 Accepted: 12 November 2021

Published online: 26 November 2021

\section{References}

1. Healthcare-associated infections in intensive care units - annual epidemiological report for 2017. European Centre for Disease Prevention and Control. http://ecdc.europa.eu/en/publications-data/healthcare-assoc iated-infections-intensive-care-units-annual-epidemiological. Accessed 2019 Oct.

2. Guerci P, Bellut H. Outcomes of Stenotrophomonas maltophilia hospitalacquired pneumonia in intensive care unit: a nationwide retrospective study. Crit Care. 2019. https://doi.org/10.1186/s13054-019-2649-5.

3. Nseir S, Di Pompeo C. Intensive care unit-acquired Stenotrophomonas maltophilia: incidence, risk factors, and outcome. Crit Care. 2006. https:// doi.org/10.1186/cc5063.

4. Saugel B, Eschermann K. Stenotrophomonas maltophilia in the respiratory tract of medical intensive care unit patients. Eur J Clin Microbiol Infect Dis. 2012. https://doi.org/10.1007/s10096-011-1459-8.

5. Pathmanathan A, Waterer GW. Significance of positive Stenotrophomonas maltophilia culture in acute respiratory tract infection. Eur Respir J. 2005. https://doi.org/10.1183/09031936.05.00096704.

6. Scholte JB, Zhou TL. Stenotrophomonas maltophilia ventilator-associated pneumonia. A retrospective matched case-control study. Infect Dis. 2016. https://doi.org/10.1080/23744235.2016.1185534

7. Hanes SD, Demirkan K. Risk factors for late-onset nosocomial pneumonia caused by Stenotrophomonas maltophilia in critically ill trauma patients. Clin Infect Dis. 2002. https://doi.org/10.1086/341022.

8. Falagas ME, Kastoris AC. Attributable mortality of Stenotrophomonas maltophilia infections: a systematic review of the literature. Future Microbiol. 2009. https://doi.org/10.2217/fmb.09.84

9. El Chakhtoura NG, Saade E. Therapies for multidrug resistant and extensively drug-resistant non-fermenting gram-negative bacteria causing nosocomial infections: a perilous journey toward "molecularly targeted" therapy. Expert Rev Anti Infect Ther. 2018. https://doi.org/10.1080/14787 210.2018.1425139.

10. Shio-Shin J, Yin-Chun C. Epidemiology, treatment, and prevention of nosocomial bacterial pneumonia. J Clin Med. 2020;9(1):275. https://doi. org/10.3390/jcm9010275.

11. Toulouse E, Masseguin C. French legal approach to clinical research. Anaesth Crit Care Pain Med. 2018. https://doi.org/10.1016/j.accpm.2018. 10.013

12. Von Elm E, Altman DG, Egger M, et al. The strengthening the reporting of observational studies in epidemiology (STROBE) statement: guidelines for reporting observational studies. Lancet. 2007;370(9596):1453-7.

13. Koenig SM, Truwit JD. Ventilator-associated pneumonia: diagnosis, treatment, and prevention. Clin Microbiol Rev. 2006;19(4):637-57. https://doi. org/10.1128/CMR.00051-05.

14. Torres A, Niederman MS. International ERS/ESICM/ESCMID/ALAT guidelines for the management of hospital-acquired pneumonia and ventilator-associated pneumonia. Eur Respir J. 2017. https://doi.org/10. 1183/13993003.00582-2017.
15. Recommandations 2019 du comité de l'antibiogramme de la société française de microbiologie. https://www.sfm-microbiologie.org/2019/01/ 07/casfm-eucast-2019/. Accessed Jan 2019.

16. Ibn Saied W, Merceron S. Ventilator-associated pneumonia due to Stenotrophomonas maltophilia: risk factors and outcome. J Infect. 2019. https:// doi.org/10.1016/j.jinf.2019.10.021.

17. Tseng CC, Fang WF. Risk factors for mortality in patients with nosocomial Stenotrophomonas maltophilia pneumonia. Infect Control Hosp Epidemiol. 2009. https://doi.org/10.1086/648455

18. Yin C, Yang W. Co-infection of Pseudomonas aeruginosa and Stenotrophomonas maltophilia in hospitalised pneumonia patients has a synergic and significant impact on clinical outcomes. Eur J Clin Microbiol Infect Dis. 2017. https://doi.org/10.1007/s10096-017-3050-4.

19. Chastre J, Wolff M. Comparison of 8 vs 15 days of antibiotic therapy for ventilator-associated pneumonia in adults: a randomized trial. JAMA. 2003 https://doi.org/10.1001/jama.290.19.2588.

20. Pugh R, Grant C. Short-course versus prolonged-course antibiotic therapy for hospital-acquired pneumonia in critically ill adults. Cochrane Database Syst Rev. 2015. https://doi.org/10.1002/14651858.CD007577.pub3.

21. Bouglé $A$, Foucrier $A$, Dupont $H$, et al. Impact of the duration of antibiotics on clinical events in patients with Pseudomonas aeruginosa ventilator-associated pneumonia: study protocol for a randomized controlled study. Trials. 2017. https://doi.org/10.1186/s13063-017-1780-3.

22. Shah MD, Coe KE. Efficacy of combination therapy versus monotherapy in the treatment of Stenotrophomonas maltophilia pneumonia. J Antimicrob Chemother. 2019. https://doi.org/10.1093/jac/dkz116.

23. $\mathrm{Ko} \mathrm{JH}$, Kang $\mathrm{Cl}$, Cornejo-Juárez P. Fluoroquinolones versus trimethoprim-sulfamethoxazole for the treatment of Stenotrophomonas maltophilia infections: a systematic review and meta-analysis. Clin Microbiol Infect. 2019. https://doi. org/10.1016/j.cmi.2018.11.008.

24. Wang YL, Scipione MR. Monotherapy with fluoroquinolone or trimethoprimsulfamethoxazole for treatment of Stenotrophomonas maltophilia infections. Antimicrob Agents Chemother. 2014. https://doi.org/10.1128/AAC.01324-13.

25. Foster RA, Troficanto C. Utility of combination antimicrobial therapy in adults with bloodstream infections due to Enterobacteriaceae and non-fermenting gram-negative bacilli based on in vitro analysis at two community hospitals. Antibiotics. 2019. https://doi.org/10.3390/antibiotics8010015.

26. Paul M, Lador A. Beta lactam antibiotic monotherapy versus beta lactamaminoglycoside antibiotic combination therapy for sepsis. Cochrane Database Syst Rev. 2014. https://doi.org/10.1002/14651858.CD003344.pub3.

27. Vardakas KZ, Tansarli GS. $\beta$-Lactam plus aminoglycoside or fluoroquinolone combination versus $\beta$-lactam monotherapy for Pseudomonas aeruginosa infections: a meta-analysis. Int J Antimicrob Agents. 2013. https://doi.org/10. 1016/j.ijantimicag.2012.12.006.

28. Dexter C, Murray GL. Community-acquired Acinetobacter baumannii: clinical characteristics, epidemiology and pathogenesis. Expert Rev Anti Infect Ther. 2015. https://doi.org/10.1586/14787210.2015.1025055.

29. Gaudet A, Martin-Loeches I. Accuracy of the clinical pulmonary infection score to differentiate ventilator-associated tracheobronchitis from ventilatorassociated pneumonia. Ann Intensive Care. 2020. https://doi.org/10.1186/ s13613-020-00721-4.

30. Kalil AC, Metersky ML. Management of adults with hospital-acquired and ventilator-associated pneumonia: 2016 clinical practice guidelines by the infectious diseases society of America and the American thoracic society. Clin Infect Dis. 2016. https://doi.org/10.1093/cid/ciw353.1717.

\section{Publisher's Note}

Springer Nature remains neutral with regard to jurisdictional claims in published maps and institutional affiliations. 